\title{
Suppression of self-pulsing instabilities in free-electron lasers using delayed optical feedback
}

\author{
Clément Evain, Christophe Szwaj, ${ }^{*}$ and Serge Bielawski \\ Laboratoire de Physique des Lasers, Atomes et Molécules, \\ Unité Mixte de Recherche No. 8523 associé au Centre National de la Recherche Scientifique, \\ Centre d'Études et de Recherches Lasers et Applications, \\ Fédération de Recherche No. 2416 associcié au Centre National de la Recherche Scientifique, \\ Université Lille 1, Sciences et Technologies, Bâtiment P5, F-59655 Villeneuve d'Ascq cedex, France \\ M.E. Couprie \\ Synchrotron SOLEIL, L'Orme des Merisiers, Saint-Aubin, Boite Postale 34, F-91192 Gif-sur-Yvette, France \\ Masahito Hosaka \\ Graduate School of Engineering, Nagoya University, Nagoya 464-8603, Japan \\ Akira Mochihashi \\ Japan Synchrotron Radiation Research Institute, Hyogo 679-5198, Japan \\ Masahiro Katoh \\ UVSOR Facility, Institute for Molecular Science, 444-8585 Okazaki, Japan, \\ and School of Physical Sciences, The Graduate University for Advanced Studies (SOKENDAI), Okazaki 444-8585, Japan
} (Received 5 January 2012; published 4 April 2012)

\begin{abstract}
We show that the macropulse instability affecting storage ring free-electron laser (FEL) oscillators can be suppressed using a delayed optical feedback. The principle, known as coherent photon seeding, consists in reinjecting a very small part of the laser output in the laser cavity in order to create a new deterministic solution. The feedback is shown to be efficient over a large range of the detuning parameter of the FEL cavity, even with very small fractions of reinjected power $\left(<10^{-8}\right.$ here from inside to inside the cavity). The experiments have been performed on the UVSOR-II storage ring free-electron laser.

DOI: 10.1103/PhysRevSTAB.15.040701

PACS numbers: 41.60.Cr, 05.45.-a, 42.65.Sf
\end{abstract}

\section{INTRODUCTION}

Free-electron lasers (FELs) are coherent light sources based on the interaction between relativistic electron bunches and a spatially periodic magnetic field [1,2]. They emit femtosecond/picosecond laser pulses synchronized with the electron bunches. In the so-called oscillator configuration, the laser pulses experience round-trips in an optical cavity, and are synchronously pumped by the electron bunches. The FEL dynamics is mainly governed by the synchronism between the optical pulses and the electron bunches either circulating in a storage ring $[3,4]$ or coming from a linear accelerator [5-8].

In storage ring FEL oscillators (SR-FELs), except near perfect synchronism, where the FEL emits stable picosecond pulses of constant amplitude, dynamical instabilities are systematically observed. As a main reason, the dynamics become hypersensitive to noise, in a way that

\footnotetext{
*christophe.szwaj@univ-lille1.fr
}

Published by the American Physical Society under the terms of the Creative Commons Attribution 3.0 License. Further distribution of this work must maintain attribution to the author(s) and the published article's title, journal citation, and DOI. is formally equivalent to the case of classical actively mode-locked lasers [9]. At small values of the detuning, the pulse train remains relatively regular, and the instability affects the internal structure of the FEL pulses [10]. At larger detunings, another instability leads to full scale modulations of the envelope of the laser pulse train. This regime, called "macropulse instability" in the case of SR-FEL, has a deterministic origin $[5,11,12]$, and shares deep similarities with classical mode-locked lasers $[9,13]$.

Those unstable regimes, either of deterministic or stochastic origin, have motivated studies about their control or suppression. In the case of the macropulse instability, it has been shown theoretically $[11,12]$ and experimentally [14-17] that the envelope can be controlled using an optoelectronic feedback. Besides, control of the internal pulse structure instabilities (at a picosecond scale) required a different type of control strategy. A particularly efficient strategy is to use an all-optical feedback technique, known in classical laser physics as coherent photon seeding [18-20]. The success of noise suppression (or increase of coherence) has been demonstrated in the case of the UVSOR SR-FEL [21].

In this paper, we focus on a surprising side effect of this all-optical feedback (originally designed to increase the 
coherence of the individual FEL pulses): the suppression of the macropulse instabilities themselves. First, we will present experimental results obtained on the UVSOR-II SR-FEL, and show, in particular, that, depending on the feedback delay, the macropulse instability domain can be entirely stabilized. Then, the stabilization process will be investigated numerically, and from the nonlinear dynamics point of view.

\section{EXPERIMENTS}

The experimental setup is displayed in Fig. 1. On the UVSOR-II SR-FEL oscillator, we applied an optical feedback using a simple plane aluminum mirror, placed at a distance of the order of $13.3 \mathrm{~m}$ (the choice of this value will

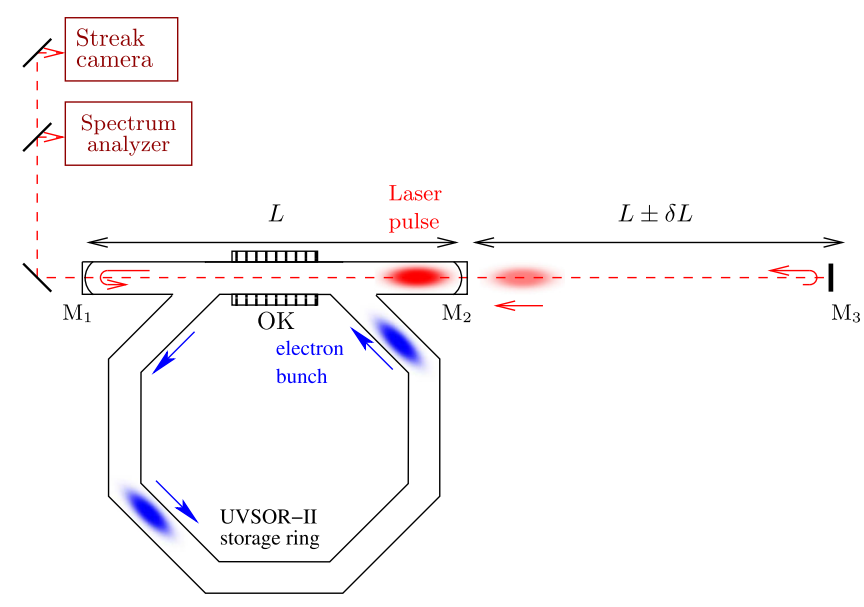

FIG. 1. Experimental setup. The feedback mirror is placed outside the FEL cavity at distance $L+\delta L$, where $L$ is the cavity length $(13.3 \mathrm{~m})$. The feedback mirror position can be changed allowing one to produce a positive or negative delay $\pm \delta L$. The other FEL output is monitored using a double sweep streak camera and a real time spectrum analyzer. OK is the optical klystron. $M_{2}$ is the output coupler (with $0.4 \%$ transmission, and $6 \mathrm{~m}$ radius of curvature). $M_{1}$ is a high reflection mirror (with $8 \mathrm{~m}$ radius of curvature). $M_{3}$ is a plane highly reflecting feedback mirror.

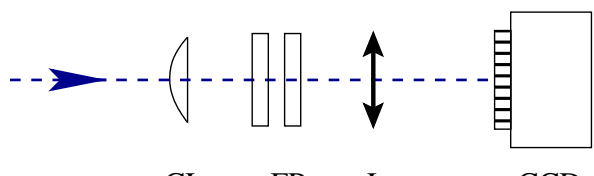

$$
\text { CL FP L } \quad \text { CCD }
$$

FIG. 2. System used for recording the temporal evolution of the optical spectrum. The laser beam is focused by a cylindrical lens (CL), $5 \mathrm{~cm}$ length, on a Fabry-Pérot interferometer (FP) EXFO TL15, used as an etalon (Finesse $>100$, spacing $0.5 \mathrm{~mm})$. The far-field interference figure is then imaged, using the lens (L) with $10 \mathrm{~cm}$ focal length onto a Perkin-Elmer LD3541 CCD line scan camera (512 pixels, 67000 lines/s). be explained below). The storage ring operates with two electron bunches, and the FEL is tuned at $420 \mathrm{~nm}$. The fast evolution of the FEL output is monitored with a double sweep streak camera (Hamamatsu C5680), allowing to record directly the pulse shape with a resolution 2 ps. In parallel, the optical spectrum is also monitored using a specially designed real time spectrum analyzer composed of a planar-planar Fabry-Pérot etalon followed by a CCD line scan camera (Fig. 2). The sweeps of the streak camera and the recordings of the CCD camera are synchronized. This allows simultaneous single shot recordings of the evolution of spectra and shapes.

The feedback is expected to have an effect when the delay $\tau_{F}$ is small (of the order of the bunch duration $\tau_{B}$ ), or near a multiple of the cavity round-trip time $\tau_{R}$, namely,

$$
\begin{gathered}
\tau_{F}=n \tau_{R}+\delta \tau \\
\text { with } \delta \tau=O\left(\tau_{B}\right) .
\end{gathered}
$$

Here we choose $n=1$, i.e., a distance of the order of $13.3 \mathrm{~m}$. Compared to the $n=0$ case, this choice allowed us to explore the effect for any sign of the delay $\delta \tau$.

From a practical point of view, adjustment of the key parameter $\delta \tau$ over a range $O\left(\tau_{B}\right)$ corresponds to the displacement of the feedback mirror position $\delta L=2 \delta \tau$ over a range of a few centimeters. A consequence is that stability of the feedback delay was not a dramatic issue (as would have been the case, e.g., if interferometric precision would have been required). Moreover, stabilization did not require the use of optics for matching the feedback mode on the laser mode. Instead, we let the laser beam freely
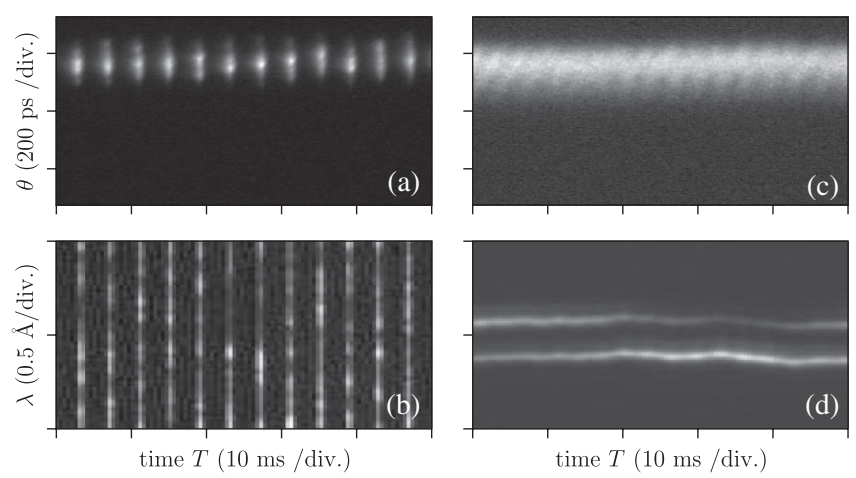

FIG. 3. Experimental suppression of the macropulse instability of SR-FEL using an optical feedback. Upper figures are double sweep streak camera images of the FEL output, lower figures are associated optical spectra. (a), (b) Macropulse instability without feedback; (c), (d) with optical feedback. The feedback delay is $\delta \tau=130$ ps (i.e., a mirror position shifted by $\delta L \approx 2 \mathrm{~cm}$ from the reference position). The fraction of reinjected power is $0.5 \times 10^{-8}$ (intracavity power reinjected inside the cavity). The rf frequency detuning is around $6 \mathrm{~Hz}$. Each streak camera recording is synchronized with its corresponding spectrum. 
diverge and diffract, leading to a beam diameter in the several $\mathrm{cm}$ range (detailed calculations are given in the Appendix). This point was of great practical interest, as it relaxed considerably the need for transverse stabilization of the feedback mirror.

A typical result showing the efficiency of the optical feedback for the suppression of the macropulse instability is displayed in Fig. 3. Figure 3(a) shows a double sweep streak camera image of the FEL output in a regime of macropulse instability. The optical spectrum, Fig. 3(b), displays a pulsed structure with a very large width.
When the optical feedback is applied, the pulsed envelope is replaced by an almost constant envelope as shown in Fig. 3(c). This stabilization of the envelope is also clearly visible in the time-resolved optical spectrum recording, displayed in Fig. 3(d) (in addition to the already known spectral narrowing [21]). Note that the presence of two horizontal lines in the optical spectrum of Fig. 3(d) may be coming from additional reflection. This is currently under investigation but beyond the scope of this paper.

To obtain information on the parameter ranges allowing control, we performed systematic recordings of the FEL

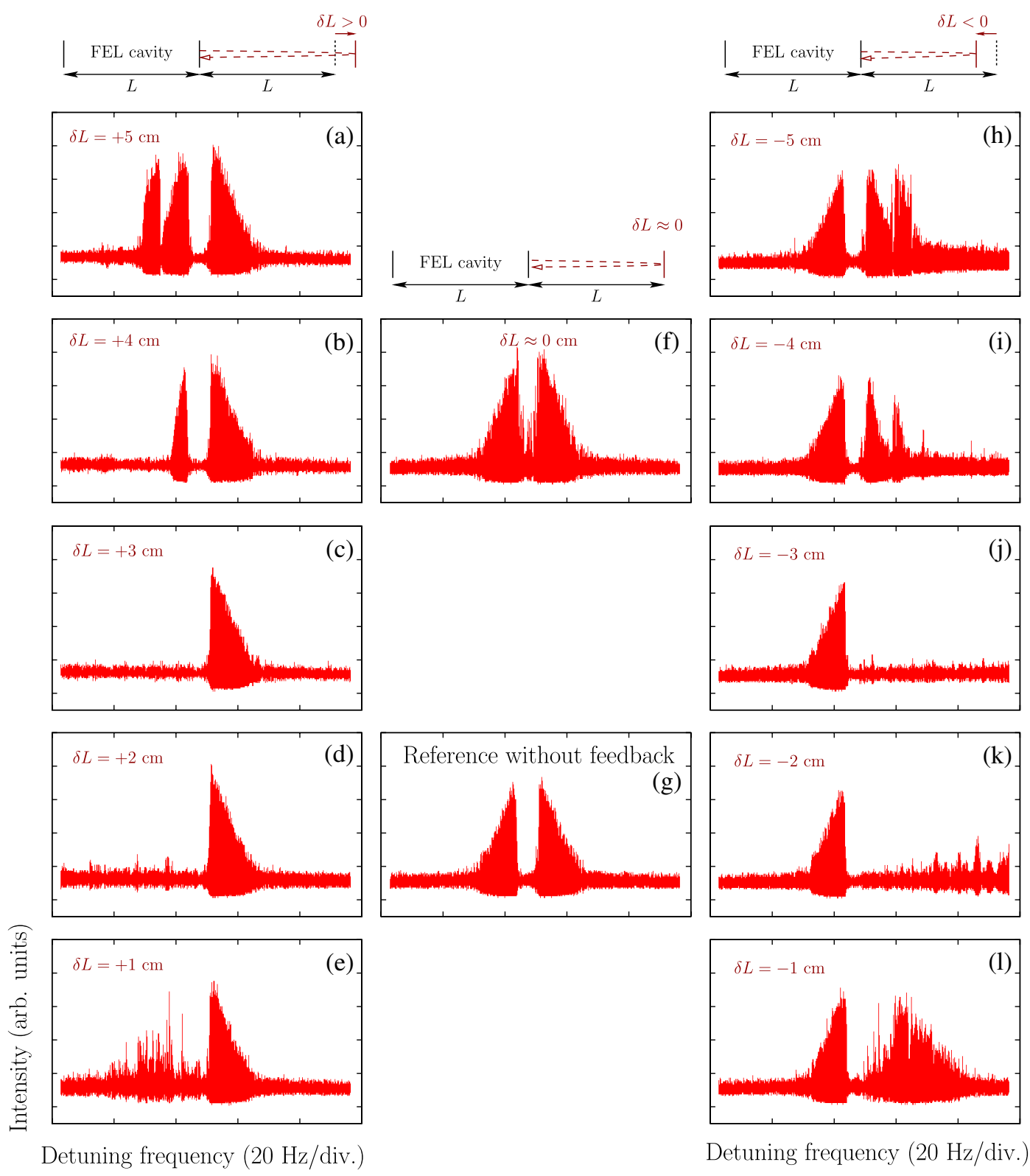

FIG. 4. Systematic investigation of bifurcation diagrams (detuning curves) of the free-electron laser versus feedback mirror position $\delta L$ (feedback delay is $\delta \tau=2 \delta L$ ). (a) $\delta L=+5 \mathrm{~cm}$; (b) $\delta L=+4 \mathrm{~cm}$; (c) $\delta L=+3 \mathrm{~cm}$; (d) $\delta L=+2 \mathrm{~cm}$; (e) $\delta L=+1 \mathrm{~cm}$; (f) $\delta L \approx 0 \mathrm{~cm}$ (defined as the value of $\delta L$ giving an approximately symmetric diagram); (g) reference curve without feedback; (h) $\delta L=-5 \mathrm{~cm}$; (i) $\delta L=-4 \mathrm{~cm}$; (j) $\delta L=-3 \mathrm{~cm}$; (k) $\delta L=-2 \mathrm{~cm}$; (l) $\delta L=-1 \mathrm{~cm}$. 
detuning curves versus feedback delay (Fig. 4). These data show that the macropulse instability can be suppressed over a large domain of $\mathrm{rf}$ detuning that can reach-for a proper choice of the delay-half the detuning curve [Figs. 4(c) and 4(j)]. In addition, by using a positive or negative relative position $\delta L$ of the mirror, it is possible to control the positive or negative domain of the detuning curve, as in the cases of Figs. 4(c) and 4(j).

The amount of feedback can be evaluated by calculating the projection of the reinjected Gaussian beam onto the FEL cavity mode, and taking into count the reflection coefficient of the output coupler. The fraction $\alpha^{2}$ of intracavity power that is reinjected on the FEL mode inside the cavity can be written as

$$
\alpha^{2}=T_{\mathrm{OC}}^{2} R_{\mathrm{FM}}|\eta|^{2},
$$

where $T_{\mathrm{OC}}$ is the transmission of the output coupler (this mirror is traversed 2 times), $R_{\mathrm{FM}}$ is the reflection coefficient of the feedback mirror, and $\eta$ is the overlap integral (scalar product) between the reinjected beam and the FEL cavity mode. Calculation of the overlap integral is detailed in the Appendix, assuming Gaussian beams with perfect alignment. The overlap factor is a small parameter $\left(|\eta|^{2} \approx 1.6 \times 10^{-3}\right)$, and the overall feedback coefficient (fraction of intracavity power reinjected inside the cavity mode) is

$$
\alpha^{2}=0.57 \times 10^{-8}
$$
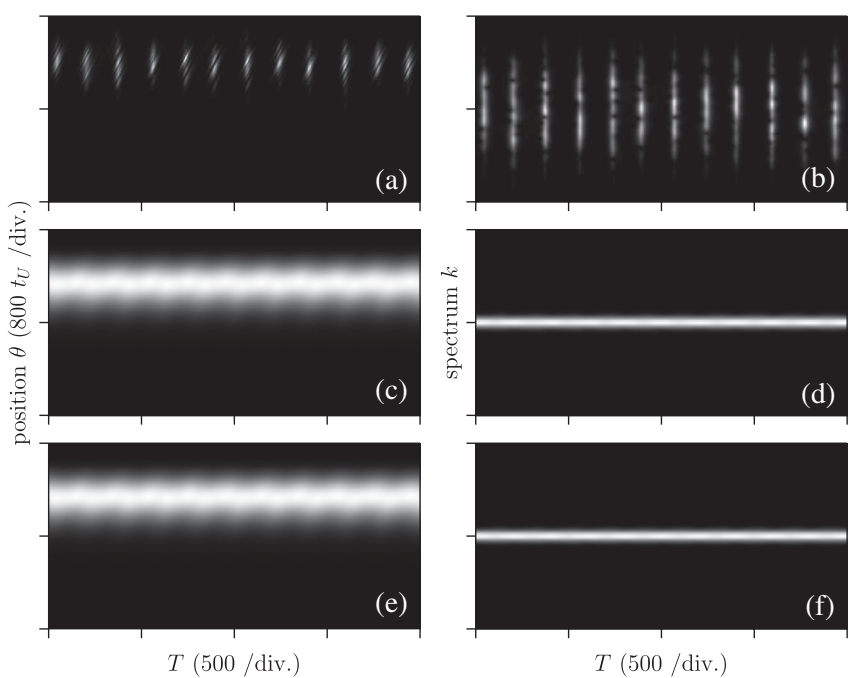

(c)

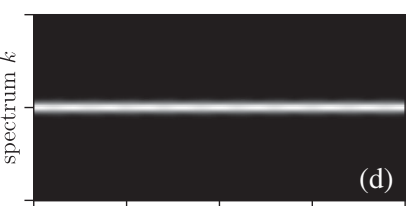

(e)

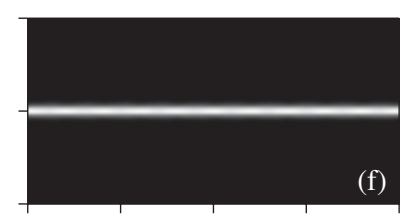

$T(500 /$ div. $)$

FIG. 5. Numerical solution of the FEL model (5). Left figures are the pulse shapes versus time $|e(\theta, T)|^{2}$, and right figures are the optical spectra $\left[|\tilde{e}(k, T)|^{2}\right.$, where $\tilde{e}(k, T)$ is the Fourier transform of $e(\theta, T)]$. The four upper figures correspond to realistic conditions: without feedback [(a) and (b)], and without feedback [(c) and (d)]. The two lower figures are reference cases (with feedback), considering an artificial condition where the spontaneous emission noise $\eta$ is taken equal to zero. Parameters are $v=6, \alpha=1 \times 10^{-4}$, and $a=600 . \eta=1 \times 10^{-14}$ for (a)-(d), and $\eta=0$ for (e) and (f).

\section{NUMERICAL RESULTS AND INTERPRETATION}

Numerical investigation of the SR-FEL dynamics may be performed using the well-known Haus master equation approach commonly used for mode-locked lasers [9,13,22-24], and established independently for FELs $[25,26]$. Haus-type models basically describe the evolution of the successive complex envelopes of field profiles $e_{n}(\theta)$ (with $\theta$ defined by $\theta=z / c$ and $z$ the longitudinal coordinate of the pulse) at each round-trip $n$. Here the series $e_{n}(\theta)$ is taken in synchronism with the electron bunch passage. We are thus in the presence of a spatiotemporal system for which the relevant space is $\theta$, and the relevant time is the round-trip number $n$. The continuous limit is taken, so that we transform the discrete time $n$ into a continuous "slow time" $T$ [22].

The evolution of $e(\theta, T)$ obeys the dimensionless equation $[9,10,21]$ :

$$
\begin{aligned}
e_{T}(\theta, T)= & -e(\theta, T)+g(T) f(\theta)\left[e(\theta, T)+e_{\theta \theta}(\theta, T)\right] \\
& -v e_{\theta}(\theta, T)+\sqrt{\eta} \xi(\theta, T)+\alpha e(\theta+a, T)
\end{aligned}
$$

where the slow time $T$ associated with the round-trips is expressed in units of the field cavity lifetime $\tau_{c}$ (typically in the microsecond range). The fast time $\theta$ resolving the pulse shape is expressed in units of $t_{u}=\pi /\left(\sqrt{2} \Delta \omega_{g}\right)$ (in the subpicosecond range) with $\Delta \omega_{g}$ the gain width of the operating line [27]. Physically the diffusion operator accounts for the finite linewidth of the gain [22]. Note that a first derivative operator of the form $\alpha g(T) f(\theta) \partial_{\theta} e[22,26]$ is also present, and accounts for the refractive index of the gain medium (lethargy) [22,26]. This latter is neglected because it is not found to affect significantly the results (only a shift of the bifurcation points in $v$ by $\alpha v$ ), for the parameters considered here. $v$ expresses the mismatch between the period of the electron bunch passages $T_{e}$ and the laser cavity round-trip time $T_{L}: v=\frac{T_{L}-T_{e}}{T_{L}} \frac{t_{U}}{\tau_{c}} \cdot v$ plays the role of an advection velocity (in units of $t_{U} / \tau_{c}$ ) and is the main control parameter of the FEL. $v$ can also be expressed in function of variation of the $\mathrm{rf}$ frequency $\delta \nu_{\mathrm{rf}}: v=\frac{\delta \nu_{\mathrm{rf}}}{\nu_{\mathrm{rf}}} \frac{\tau_{c}}{t_{U}}$.

The gain shape due to the electron bunch longitudinal distribution is characterized by $f(\theta)=\exp \left(-\theta^{2} / 2 \sigma_{b}^{2}\right)$, where $\sigma_{b}$ is the bunch duration in units of $t_{u}$. The effect of spontaneous emission is taken into account by the white noise term $\xi(\theta, T)$ with $\left\langle\xi^{*}\left(\theta^{\prime}, T^{\prime}\right) \xi(\theta, T)\right\rangle=\delta\left(\theta-\theta^{\prime}\right) \times$ $\delta\left(T-T^{\prime}\right)$. The level of noise is controlled by the parameter $\eta$, which is purely phenomenological. The gain dynamics $g(T)$ depends on the precise accelerator and insertion device (undulator system). A simple expression for a storage ring can be written in dimensionless units as $[8,28]$

$$
g(T)=\frac{A}{\sigma(T)} \exp \left\{-\left[\sigma^{2}(T)-1\right] / 2\right\}
$$




$$
\text { with } \frac{d \sigma^{2}}{d T}=\frac{1}{T_{s}}\left(1-\sigma^{2}+\int_{0}^{L}|e(\theta, T)|^{2} d \theta\right)
$$

Equation (6) links the gain $g$ to the energy spread $\sigma$, which is the rms width of the electron energy distribution in units of its value without laser emission. $A$ is the maximum gain in units of the cavity losses (laser operation requires $A>1$ ). Equation (7) accounts for the relaxation of $\sigma^{2}$, and the bunch heating by the laser which provides the gain saturation process. The heating relaxation time $T_{s}$ is equal to the synchrotron damping time in units of the field cavity lifetime $\left(T_{s} \gg 1\right)$. $L$ is the round-trip time in units of $t_{u}$.

The feedback is applied through the term $\alpha e(\theta+a, T)$, with $\alpha$ and $a$ the gain and the feedback delay (shift parameter), respectively. In the following we use typical parameters for the UVSOR-II FEL: $1 / T s=0.0047, \sigma_{b}=$ $862, A=4.5, t_{u}=80 \mathrm{fs}, \eta=1 \times 10^{-14}$. The unit of $T$ is $\tau_{c}=40 \mu \mathrm{s}$

Numerical integration of the model (5) allows one to study the effect of noise on the feedback process. Figure 5(a) displays the temporal evolution of the field amplitude and Fig. 5(b) its associated spectrum in the "pulsed" regime without feedback and in the presence of noise. When feedback is applied, the pulsed regime can be suppressed and is replaced by an almost constant amplitude regime with a very narrow spectrum, as illustrated on Figs. 5(c) and 5(d). These results are in good agreement with the experimental findings.

As spontaneous noise is known to be an important ingredient of the FEL dynamics, we have also integrated the FEL equations in conditions where the noise $\eta$ is equal to zero. Indeed, although nonphysical, the results in this reference case provide important information on the dynamical processes involved. In Figs. 5(e) and 5(f) is displayed this reference case (without noise) with the same parameters as for Figs. 5(c) and 5(d).

At first view, the result may seem trivial, because the two cases (with and without noise) give almost exactly the same results. However, this behavior strongly contrasts with the FEL behavior in usual conditions (i.e., without feedback), as its behavior is well known to be extremely sensitive to the spontaneous emission noise [21]. More precisely, without noise we would observe-instead of a noisy pulsing laser [Figs. 5(a) and 5(b)]—an FEL in the "OFF" state (zero output power).
Therefore, the interpretation from the nonlinear dynamics point of view can be summarized by the following three points. The feedback creates a solution that is: (i) new (i.e., do not exist without feedback), (ii) deterministic [since it remains when noise is set to zero, Figs. 5(e) and 5(f)], (iii) and robust to noise, as (in contrast to the FEL without feedback).

Moreover, for proper feedback delay, we emphasize that the amount of feedback can be extremely small, the condition being that the reinjected signal is larger that the noise level.

\section{CONCLUSION}

In conclusion, we showed experimentally and numerically that the macropulse instability of the storage-ring free-electron laser oscillator can be suppressed using a "simple" optical feedback. For adequate feedback delay, the amount of feedback necessary suppressing the instability can be extremely small (of the order of $1 \times 10^{-8}$ from inside-to-inside the cavity in the experiments). In addition, we evidenced numerically that this feedback process is characterized by the creation of a new steady state solution that is deterministic.

\section{ACKNOWLEDGMENTS}

This work was supported by the Joint Studies Program of the Institute for Molecular Science, the JSPS program for research in Japan, and the ANR (DYNACO project). The CERLA is supported by the French Ministère chargé de la Recherche, the Région Nord-Pas de Calais, and the FEDER.

\section{APPENDIX: CALCULATION OF THE FRACTION OF POWER REINJECTED IN THE CAVITY}

To evaluate the amount of reinjected power, we calculate the overlap integral between the following two Gaussian beams at the same location (see Fig. 6): (i) The laser mode (inside the FEL cavity) — we denote $q^{-}$the complex curvature radius of the mode just after reflection on the output coupler $M_{2}$; (ii) the Gaussian beam provided by the feedback mirror, just after passing through $M_{2}$ (i.e., inside the FEL cavity) - we denote $q_{f}^{-}$the corresponding complex radius of curvature.

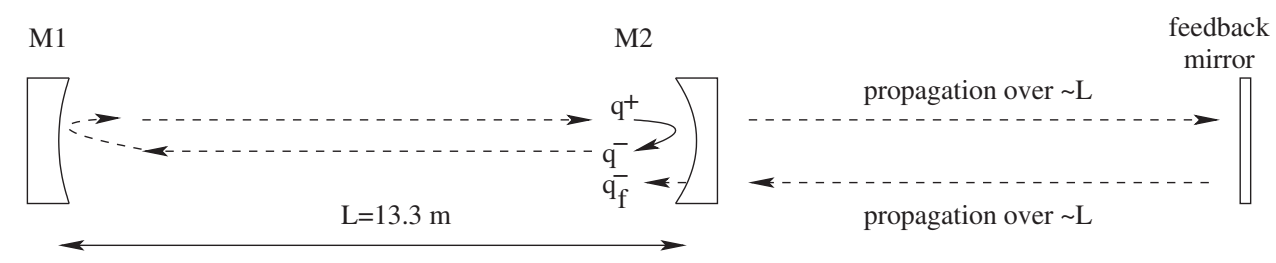

FIG. 6. Illustration of the definitions of the complex curvature radii involved in the feedback coefficient calculations. All radii are calculated at the same location: the location of the output coupler $M_{2}$, inside the cavity, and leftwards. $q^{-}$correspond to the lasing mode. The feedback beam eventually reinjected and has a complex curvature radius $q_{f}^{-}$. 
Assuming perfect alignment, the fraction of power reinjected by the feedback into the laser mode (from inside to inside) is

$$
\alpha^{2}=T_{\mathrm{OC}}^{2} R_{\mathrm{FM}}|\eta|^{2}\left(q^{-}, q_{f}^{-}\right),
$$

with $T_{\mathrm{OC}}$ the transmission of the output coupler $M_{2}, R_{\mathrm{FM}}$ the reflection coefficient of the feedback mirror. $\eta$ is the overlap integral defined in a general way by

$$
|\eta|^{2}\left(q_{1}, q_{2}\right)=\frac{\left|\int_{x=-\infty}^{x=+\infty} \int_{y=-\infty}^{y=+\infty} \phi_{q_{1}}^{*}(x, y) \phi_{q_{2}}(x, y) d x d y\right|^{2}}{\int_{x=-\infty}^{x=+\infty} \int_{y=-\infty}^{y=+\infty}\left|\phi_{q_{1}}(x, y)\right|^{2} d x d y \int_{x=-\infty}^{x=+\infty} \int_{y=-\infty}^{y=+\infty}\left|\phi_{q_{2}}(x, y)\right|^{2} d x d y},
$$

where $\phi_{q 1}$ and $\phi_{q_{2}}$ are the (unnormalized) Gaussian beam transverse profile defined by

$$
\phi_{q}(x, y)=e^{-\left[i k\left(x^{2}+y^{2}\right) / 2 q\right]} .
$$

The overlap integral may be written in a simple form:

$$
|\eta|^{2}=4 \frac{z_{R 1} z_{R 2}}{\left(z_{1}-z_{2}\right)^{2}+\left(z_{R 1}+z_{R 2}\right)^{2}},
$$

where $\left(z_{1}-z_{2}\right)$ is the algebraic distance between the two waists, and $z_{R 1}, z_{R 1}$ are the Rayleigh lengths of the two beams (we have $q_{i}=z_{i}+i z_{R i}$ ).

Hence, we have

$$
\alpha^{2}=4 \frac{z_{R 1} z_{R 2}}{\left(z_{1}-z_{2}\right)^{2}+\left(z_{R 1}+z_{R 2}\right)^{2}} T_{\mathrm{OC}}^{2} R_{\mathrm{FM}}
$$

From the parameters of the cavity, we deduce

$$
q^{-}=(-6+1.51 i) \mathrm{m},
$$

and the complex curvature radius of the intracavity laser mode just before reflection on $M_{2}$ :

$$
q^{+}=(6+1.51 i) \mathrm{m} \text {. }
$$

$q_{f}^{-}$is deduced from $q^{+}$taking into account the traverse of the divergent lens (focal length $-12 \mathrm{~m}$ ) formed by $M_{2}$, the free propagation $(2 \times 13.3 \mathrm{~m})$, and the second traverse of $M_{2}$ :

$$
q_{f}^{-}=(8.61+0.055 i) \mathrm{m} .
$$

Substituting $q_{1}$ and $q_{2}$ by $q_{f}^{-}$and $q^{+}$in Eq. (A4), we can now calculate the value of the overlap integral: $|\eta|^{2}=$ 0.00164 , and the values of feedback coefficient: $\alpha^{2}=$ $T_{\mathrm{OC}}^{2} R_{\mathrm{FM}}|\eta|^{2}=0.565 \times 10^{-8}$.

[1] D. A. G. Deacon, L. R. Elias, J. M. J. Madey, G. J. Ramian, H. A. Schwettman, and T. I. Smith, Phys. Rev. Lett. 38, 892 (1977).

[2] M. Billardon, P. Elleaume, J. M. Ortega, C. Bazin, M. Bergher, M. Velghe, Y. Petroff, D. A. G. Deacon, K.E. Robinson, and J. M. J. Madey, Phys. Rev. Lett. 51, 1652 (1983).

[3] D. A. Jaroszynski, R. J. Bakker, A. F. G. van der Meer, D. Oepts, and P. W. van Amersfoort, Phys. Rev. Lett. 70, 3412 (1993).
[4] O. G. Calderón, T. Kimura, and T. I. Smith, Phys. Rev. ST Accel. Beams 3, 090701 (2000).

[5] M. Billardon, D. Garzella, and M. E. Couprie, Phys. Rev. Lett. 69, 2368 (1992).

[6] H. Hama, K. Kimura, J. Yamazaki, S. Takano, T. Kinoshita, and M.-E. Couprie, Nucl. Instrum. Methods Phys. Res., Sect. A 375, 32 (1996).

[7] V. Litvinenko, S. Park, I. Pinayev, and Y. Wu, Nucl. Instrum. Methods Phys. Res., Sect. A 475, 240 (2001).

[8] G. De Ninno, D. Fanelli, C. Bruni, and M. E. Couprie, Eur. Phys. J. D 22, 269 (2003).

[9] C. Bruni, T. Legrand, C. Szwaj, S. Bielawski, and M.E. Couprie, Phys. Rev. A 84, 063804 (2011).

[10] S. Bielawski, C. Szwaj, C. Bruni, D. Garzella, G. L. Orlandi, and M.E. Couprie, Phys. Rev. Lett. 95, 034801 (2005).

[11] G. De Ninno and D. Fanelli, Phys. Rev. E 70, 016503 (2004).

[12] G. De Ninno and D. Fanelli, Phys. Rev. Lett. 92, 094801 (2004).

[13] J. B. Geddes, W. J. Firth, and K. Black, SIAM J. Appl. Dyn. Syst. 2, 647 (2003).

[14] G. De Ninno, A. Antoniazzi, B. Diviacco, D. Fanelli, L. Giannessi, R. Meucci, and M. Trovó, Phys. Rev. E 71, 066504 (2005).

[15] C. Bruni, M.-E. Couprie, D. Garzella, G. Lambert, G.-L. Orlandi, M. Danailov, G. D. Ninno, B. Diviacco, M. Trovò, L. Giannessi, E. Allaria, R. Meucci, D. Fanelli, and S. Bielawski, in Proceedings of the 9th European Particle Accelerator Conference, Lucerne, 2004 (EPS-AG, Lucerne, 2004), p. 381.

[16] M. Couprie, C. Bruni, G. Orlandi, D. Garzella, and S. Bielawski, Nucl. Instrum. Methods Phys. Res., Sect. A 528, 263 (2004).

[17] C. Szwaj, S. Bielawski, M.-E. Couprie, C. Bruni, M. Hosaka, A. Mochihashi, and M. Katoh, Eur. Phys. J. D 59, 451 (2010).

[18] P. Beaud, J. Bi, W. Hodel, and H. Weber, Opt. Commun. 80, 31 (1990).

[19] J. Bi, W. Hodel, and H. Weber, Opt. Commun. 81, 408 (1991).

[20] G. H. C. New, Opt. Lett. 15, 1306 (1990).

[21] C. Evain, C. Szwaj, S. Bielawski, M. Hosaka, A. Mochihashi, M. Katoh, and M.-E. Couprie, Phys. Rev. Lett. 102, 134501 (2009).

[22] H. A. Haus, IEEE J. Quantum Electron. 11, 323 (1975).

[23] A. M. Dunlop, W. J. Firth, D. R. Heatley, and E. M. Wright, Opt. Lett. 21, 770 (1996). 
[24] N. Joly and S. Bielawski, Opt. Lett. 26, 692 (2001).

[25] P. Elleaume, IEEE J. Quantum Electron. 21, 1012 (1985).

[26] G. Dattoli, T. Hermsen, A. Renieri, A. Torre, and J.C. Gallardo, Phys. Rev. A 37, 4326 (1988).
[27] More precisely, the spontaneous emission spectrum and the gain spectrum of an optical klystron are typically oscillatory. $\Delta \omega_{g}$ is half the period of this oscillation.

[28] S. Bielawski, C. Bruni, G. L. Orlandi, D. Garzella, and M. E. Couprie, Phys. Rev. E 69, 045502 (2004). 